\title{
Novel characteristics of normal supraspinatus insertion in rats: an ultrastructural analysis using three-dimensional reconstruction using focused ion beam/scanning electron microscope tomography
}

\author{
Tomonoshin Kanazawa ${ }^{1,2}$ \\ Masafumi Gotoh ${ }^{2}$ \\ Keisuke Ohta ${ }^{1}$ \\ Naoto Shiba ${ }^{2}$ \\ Kei-ichiro Nakamura1
}

1 Division of Microscopic and Development Anatomy, Department of Anatomy, Kurume University School of Medicine, Fukuoka, Japan

2 Department of Orthopaedic Surgery, Kurume University School of Medicine, Fukuoka, Japan

Corresponding author:

Tomonoshin Kanazawa

Division of Microscopic and Development Anatomy, Department of Anatomy

Kurume University School of Medicine

67 Asahi-machi, Kurume City, Fukuoka, Japan

E-mail: tomochan@med.kurume-u.ac.jp

\section{Summary}

Background: the histological architecture of the insertion after a rotator cuff repair is completely different from that of normal tendon-bone insertions. Analysis of normal insertions by electron microscopy may enhance the understanding of the pathophysiology of tendon-to-bone healing after rotator cuff repair. The present study examined the normal supraspinatus insertion in rats using a new three-dimensional (3D) electron microscopic method, focused ion beam/scanning electron microscope (FIB/SEM) tomography.

Methods: normal supraspinatus insertion of adult Sprague-Dawley rats was analyzed. FIB/SEM tomography was performed on the entire insertion. The obtained serial images were reconstructed, and the 3D cellular morphology and organization of collagen bundles was observed.

Results: the cellular shapes between the tendoncartilage interface were successfully reconstructed. The cells in the cartilage region were spherical without any cellular processes, while the cells in the intermediate region had some cellular processes oriented longitudinally along the collagen bundles. In addition, these 2 regions were smoothly transferred under ultrastructural resolution.
Conclusions: structures at the normal insertion gradually changed from the fibrous cartilage to the tendon midsubstance, which may contribute to the biomechanical strength of the site. These novel cell characteristics may provide necessary knowledge for better regeneration of tendon-tobone insertions after rotator cuff repair.

KEY WORDS: electron microscope tomography, enthesis, normal supraspinatus insertion, rotator cuff, ultrastructural analysis.

\section{Introduction}

To obtain a successful outcome after rotator cuff repair, the repaired tendon needs to be anchored securely to the bone. The postoperative tendon-bone interface is mechanically weak ${ }^{1}$ and the histological architecture of the repaired site, which is termed an "indirect insertion," is completely different from that of highly differentiated, normal tendon-bone insertions. At this repaired point, the linkage between the tendon and bone is directly integrated without a fibrocartilage layer. In contrast, the normal tendon-bone insertion consists of a 4-layered structure: tendon, fibrocartilage, mineralized fibrocartilage, and bone ${ }^{2,3}$. This morphological alteration may contribute to the observed functional instability after surgical repair ${ }^{4}$.

To address this issue, a detailed structural understanding of normal tendon-bone insertions is necessary, especially in the fibrocartilage layers that mechanically connect the tendons and bones. Several researchers have studied the structure/development of normal tendon-bone insertions ${ }^{5-10}$. Galatz et al. have reported that various factors (e.g., those directing the production of the extracellular matrix and growth factors) are expressed during tendon-bone insertion development, and these factors play an important role in cartilage formation at the site. Previous histological analyses have been well performed using microscopy, but electron microscopy has not been used thus far. Electron microscopy may provide a detailed structural analysis of the tendon-bone insertion, and the information obtained may enhance the understanding of pathophysiological insertions. However, few studies have observed the tendon-bone insertion using electron microscopy.

Recently, a new three-dimensional (3D) analytical scanning electron microscopic method, namely, focused ion beam/scanning electron microscope tomography (FIB/SEM tomography), has been devel- 
oped ${ }^{11,12}$. This method enables 3D structure analysis of biological tissue with a wider range and higher resolution. Consequently, the detailed architecture of the cells and collagen bundles can be evaluated at the tendon-bone insertion using this method. In the present study, FIB/SEM tomography was used to analyze the ultrastructure of the normal supraspinatus tendon insertion in rats, which have been used as a rotator cuff tear model ${ }^{13}$. The results showed that a novel structure is formed between fibrous cartilage and tendon midsubstance, where the mechanical strength of the tendon-bone insertion is concentrated.

\section{Materials and methods}

\section{Study design}

All animals were ethically conducted according to the international standards ${ }^{14}$, and ethical approval for these studies was obtained from our animal care center. The supraspinatus tendon-humerus complex of adult Sprague-Dawley rats (weight, 510-550 g) was used as a model of normal tendon-bone insertion. FIB/SEM tomography was performed from the humerus to the supraspinatus tendon area after decalcification and embedding of the Epoxy resin (Fig. 1). The morphology of the cells and the collagen bundles at the normal tendon-bone insertion sites were reconstructed into $3 \mathrm{D}$ structures using ultrastructural resolution and were investigated.

\section{Specimen preparation}

\section{Hematoxylin and Eosin staining}

The supraspinatus humerus complex were harvested and immediately fixed in neutral buffered $10 \%$ forma-

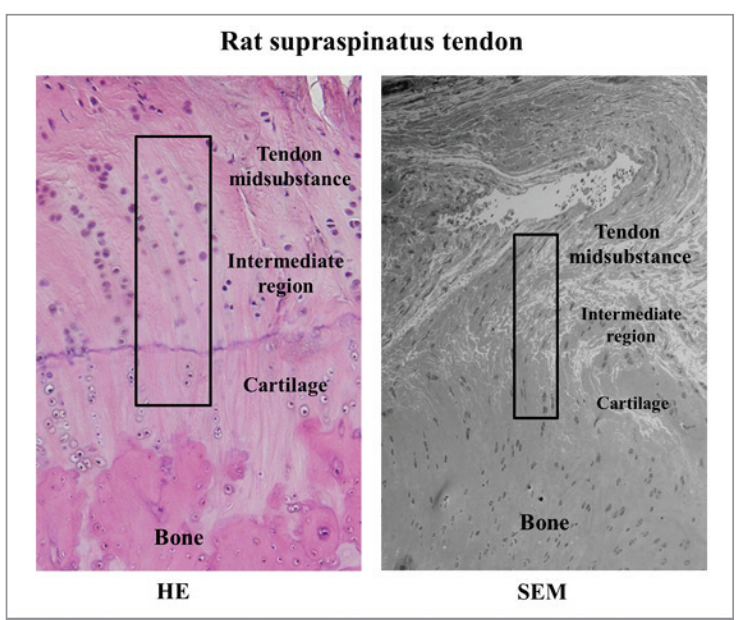

Figure 1. Analysis area. The square shows the insertion area analyzed by focused ion beam/scanning electron microscope tomography.

$\mathrm{HE}$ : hematoxylinand eosin staining SEM: scanning electron microscopy. lin for 48 hours. The specimens were decalcified in formic acid $(29 \mathrm{~g}$ citric acid, $18 \mathrm{~g}$ trisodium citrate dehydrate and $100 \mathrm{ml}$ formic acid, with distilled water added to yield a total volume of $1000 \mathrm{ml}$ ), dehydrated and embedded in paraffin. Longitudinal 5 um thick sections of the supraspinatus insertion were made. Hematoxylin and Eosin were used to stain the sections, which were examined under optical light microscopy.

\section{FIB/SEM tomography}

Sprague-Dawley rats were deeply anesthetized with diethyl ether and sodium pentobarbital, transcardially perfused through the left ventricle with heparin-containing saline, and subsequently fixed with half Karnovsky solution (2\% paraformaldehyde, $2.5 \%$ glutaraldehyde, and $2 \mathrm{mM} \mathrm{CaCl}_{2}$ in $0.1 \mathrm{M}$ cacodylate buffer). The specimens were also stained using hematoxylin and eosin. After perfusion, the supraspinatus tendon-humerus complexes were harvested and further immersed in the same fixative for $2 \mathrm{~h}$ at $4^{\circ} \mathrm{C}$. After decalcification with $5 \%$ EDTA solution for 4 weeks, the specimens were cut into small cubes and further fixed with ferrocyanate and $1 \% \mathrm{OsO}_{4}$. Subsequently, the specimens were treated with $1 \%$ thiocarbohydrazide and then immersed in a $1 \% \mathrm{OsO}_{4}$ solution. Foren blocstaining, the specimens were immersed in a solution of $4 \%$ uranyl acetate solution overnight and finally washed with distilled water. The specimens were further stained with Walton's lead aspartate solution. They were then dehydrated with a graded ethanol series, infiltrated with an epoxy resin mixture, and polymerized at $60^{\circ} \mathrm{C}$ for $72 \mathrm{~h}^{12}$. The surfaces of the embedded specimens were exposed with a diamond knife, and the resin blocks were trimmed and placed on an appropriate holder.

\section{FIB/SEM observation}

Each specimen, mounted in a special holder, was set on the stage of the FIB/SEM machinery (FEI) (Fig. 2). Serial images of the block face were acquired by repeated cycles of sample surface milling using a focused gallium ion beam (milling step: $60 \mathrm{~nm}, 1000$ cycles) and by image acquisition using SEM as a compositional contrast image from back scattered electrons (landing energy, $3.5 \mathrm{keV}$ ). The reconstructed images covered the area from the humerus to the supraspinatus tendon. In total, 3600 block face images were obtained per specimen.

\section{Histological evaluation and 3D-structure recon- struction}

The humerus to the supraspinatus tendon region was divided into 3 areas (cartilage region, intermediate region between the cartilage and tendon midsubstance, and tendon midsubstance region). In each region, the morphology of the cells and collagen bundles were 
evaluated after 3D reconstruction using Avizo 6.3 software (VSG Inc., Bordeaux, France). The 3D morphology of the cells was extracted using a semi-manual procedure. Briefly, all the image stacks were normalized using a histogram-based image filter, and a median filter was then applied to prepare the threshold segmentation. The cellular regions were then selected using the threshold method. Irregular regions in the selected regions were manually removed.

\section{Results}

We used the new FIB/SEM tomography method to identify the layer-specific cell shape within the normal supraspinatus tendon insertion, from the cartilage to tendon midsubstance regions (Figs. 1, 2).

\section{Cartilage region}

Light microscope photographs showed colonies of spherical cells in the smooth cartilaginous matrix. Electron photomicrographs showed that the matrix consisted of non-oriented delicate fibrils, and each cell existed in a lacuna with a large nucleus, abundant cytoplasm, and plasma membrane (Fig. 3). The plasma membranes were often invaginated with coated vesicles. These features indicate that the cells have typical properties of chondrocytes. The cross-
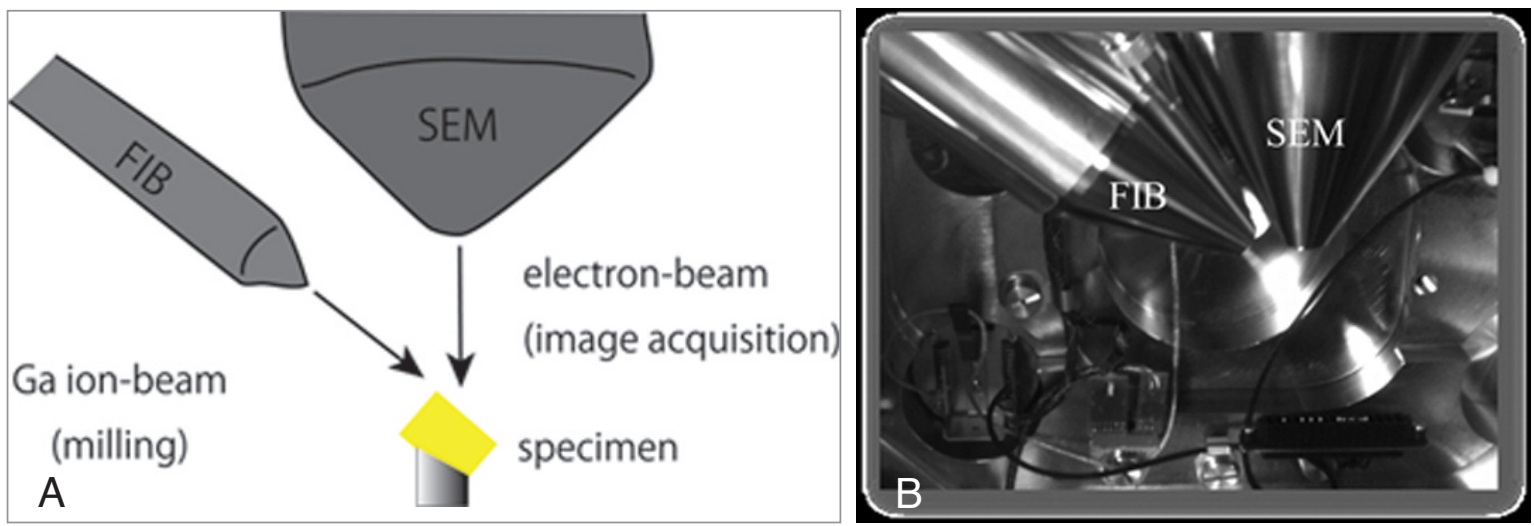

Figure 2 A-B. Focused ion beam/scanning electron microscope (FIB/SEM) tomography apparatus. Schematic (A) and photograph (B) of the FIB/SEM tomography apparatus.

3D Reconstruction

Figure 3 A-I. Micro- and ultramicrophotographs, as well as 3-dimensional (3D) reconstruction images of the cells in the normal supraspinatus insertion. Cartilage $(\mathbf{A}, \mathbf{D}, \mathbf{G})$, intermediate region $(\mathbf{B}, \mathbf{E}$, $\mathbf{H})$, tendon midsubstance $(\mathbf{C}, \mathbf{F}, \mathbf{I})$, and a cell-to-cell connection $(\mathbf{F}$, inset, red circle).

The black arrow indicates a cell process in the intermediate region. $\mathrm{HE}$ : hematoxylinand eosin staining; SEM: scanning electron microscope;

blue: cell nucleus; green: cell cytoplasm. 
sectional 3D tissue reconstruction revealed that the cells had an almost round, ellipsoidal shape, without any processes or cell-to-cell connections.

\section{Intermediate region between the cartilage and tendon midsubstance}

Light microscopic structural features of this area were almost the same as the observed in the cartilage area describe above. Electron photomicrographs showed that the cells existed in lacunae, and that they were separated from one another by microfibrils. The cell bodies were spherical, similar to those in the cartilage. However, some cellular processes were occasionally observed on the cell bodies (Fig. 3: black arrow). The 3D reconstructed images showed that each cell had a few processes, which were oriented in the same direction as the adjacent collagen bundles. However, the number of the processes was fewer than that in the tendon midsubstance region (see below), and no cell-to-cell connections via the processes were observed.

\section{Tendon midsubstance region}

Hematoxylin and eosin photomicrographs show many spindle-shaped cells located between the well-developed collagen bundles. The electron microphotographs showed that each of these cells had fibroblastic characteristics, including a large nucleus and abundant rough endoplasmic reticulum within the cytoplasm. Further, these cells had well-developed cellular processes extending from the cell bodies. The $3 \mathrm{D}$ reconstructed images from the FIB/SEM observation showed several cell processes oriented in the same direction as the adjacent collagen bundles. In addition, occasional connections between the cells, via these processes, were also observed (Fig. 4).
A summary of the cell morphologies within each layer are summarized in Table 1.

\section{Discussion}

Previous studies have characterized the point of the tendon-bone insertion as a 4-layered structure: tendon, fibrocartilage, mineralized fibrocartilage, and bone $^{2,3}$. However, these studies have not revealed ultrastructural level details. The present study used a FIB/SEM tomography technique ${ }^{11,12,15}$ to address this issue and showed the cell morphologies present in the overall tendon-cartilage interface within the supraspinatus insertion area (Fig. 5). We also successfully demonstrated the ultrastructural characteristics of the tendon-cartilage interface using 3D reconstruction. The cells in the intermediate region of tendon-cartilage displayed a chondrocyte-like morphology, but had some processes that were longitudinally oriented along the collagen bundles, unlike the typical cartilage region. These results strongly suggest that these cells are intermediate between chondrocytes and fibroblastic cells. In the tendon midsubstance region, cell processes were also longitudinally oriented along the collagen bundles, and cellular connections via processes were occasionally observed. We consider that the tendon-cartilage interface cells adapt their form during the transition from the bone to tendon to possibly contribute to the biomechanical stability at the site.

Our findings are in line with those of previous studies that reported smooth changes in the mineral distribution at the tendon-bone insertion site ${ }^{9,16}$. Schneider suggested that the fibrocartilage between tendons and bones is associated, not only with the biomechanical strength of the insertion, but also the soft-tissue elasticity during motion ${ }^{8}$. Messner concluded that the interposition of a fibrocartilaginous zone at the insertion site diminishes sudden changes in stiffness
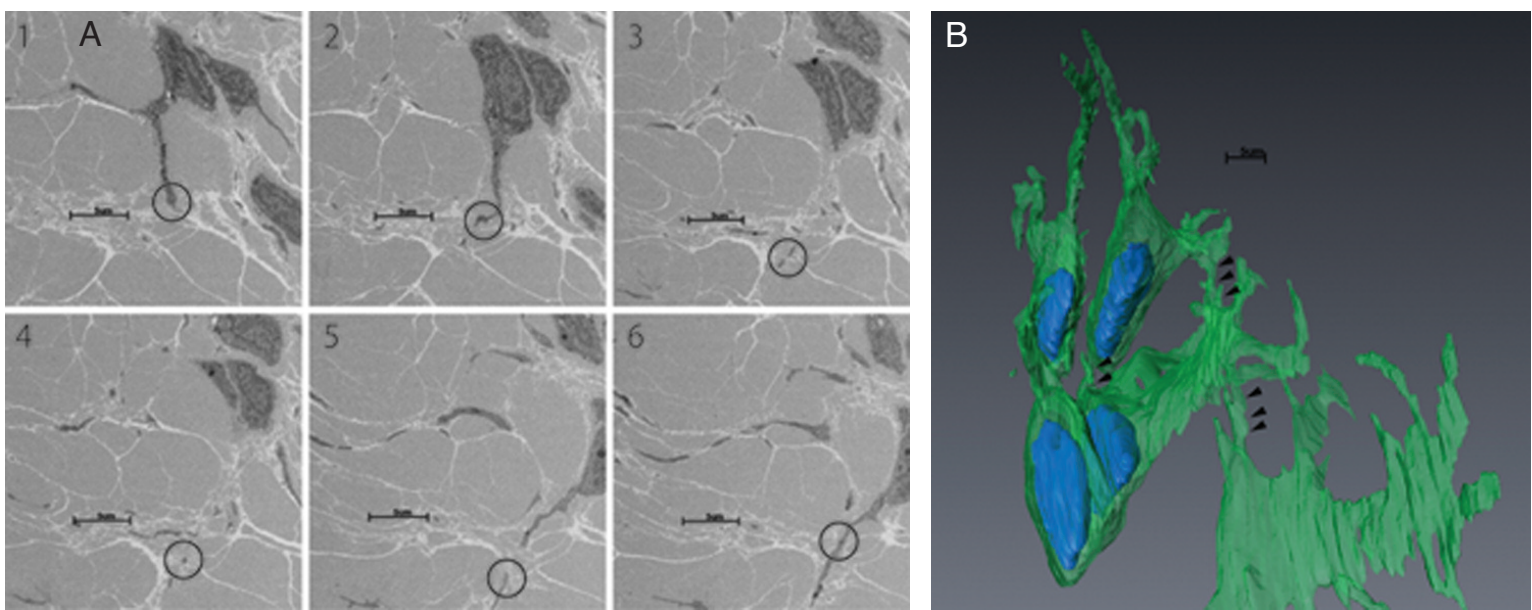

Figure 4 A-B. Cell-to-cell connections in the tendon midsubstance area. (A) Continuous block face images. The black circles indicate the process of a cell connecting to another cell. (B) 3D reconstruction image. The black arrows indicate cell-to-cell connection via the cell processes. Green: process/cell cytoplasm; blue: cell nucleus. 
Table 1. Summary of cell morphologies in the normal supraspinatus insertion.

\begin{tabular}{|c|c|c|c|c|c|}
\hline Cell area & Morphology of the cell & Cell processes & $\begin{array}{c}\text { Cell processes of the adjacent } \\
\text { collagen bundle }\end{array}$ & Porality & Cell-to-cell conn ection \\
\hline Tendon midsubstance region & Spindle shaped & Abundant & Parallel & tendon & Occasionally \\
\hline Intermediate region & Sphere shaped & Moderate & Parallel & tendon & None \\
\hline Cartilage region & Sphere shaped & None & None & None & None \\
\hline
\end{tabular}

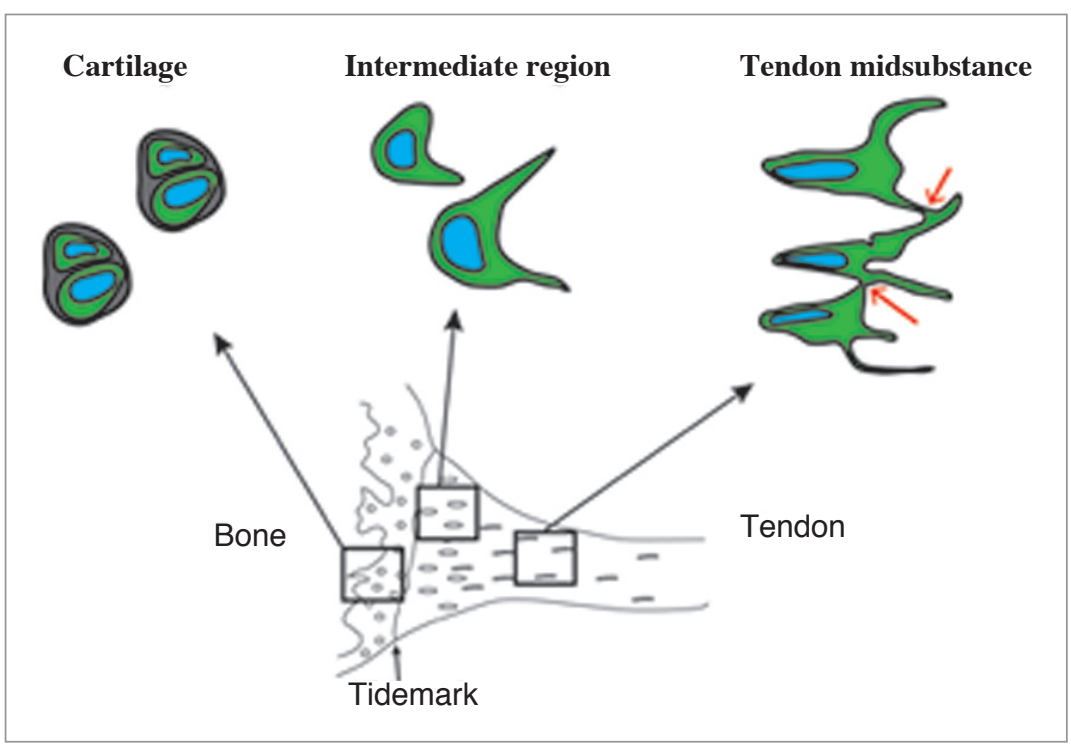

Figure 5. Schemainthe overall normal supraspinatus insertion.

The tendon-cartilage interface cells adapt their form during the transition from the bone to tendon.

between tendons/ligaments and the bone ${ }^{7}$. These findings consistently indicate that fibrocartilage acts as a stress absorber by reducing the stiffness between the soft and hard tissues, thus supporting our above mentioned hypothesis. In this context, smooth changes at the tendon-cartilage interface are a favorable feature for mechanical absorbing properties.

On the other hand, repaired tendons are directly anchored to the bones via perforating fibers ("indirect insertion") and lack mineralized and un-mineralized fibrocartilage layers ${ }^{1,17,18}$. Previous studies have attempted to enhance tendon-bone healing using various agents in acute rotator cuff repair models ${ }^{19,20}$ However, these studies have failed to recreate normal insertion fibrocartilage consisting of mineralized and un-mineralized layers. In the normal supraspinatus insertion, we discovered a cell type ultrastructurally intermediate between chondrocytes and fibroblastic cells within the tendon-cartilage interface, and we suggest that these differentiated cells may be associated with the biomechanical stability of the insertion area. Therefore, these findings suggest that optimal rotator cuff repair may require regeneration of a continuous and smooth tendon-cartilage interface, rather than reconstruction of a clear 4-layered structure.

Recent clinical results after rotator cuff repair have been favorable. However, the return rate is not acceptable after throwing disorder cuff repairs ${ }^{21}$. As stated previously, the normal tendon-bone insertion has 4-layered structures, whereas an injured insertion displays an "indirect insertion," without fibrocartilage consisting of mineralized and un-mineralized layers ${ }^{1,17,18}$. The fibrocartilage between the tendon and bone is responsible for strength, stiffness, and flexibility at the insertion $7,8,10$. These results may explain why "indirect insertion" after rotator cuff repair is acceptable for the lower-demands of middle-aged and elderly patients, but not for the higher-demands of younger patients with throwing disorders.

The morphology of the cells in the intermediate region was similar to that of chondrocytes, but the cells also had some cellular processes. Studies have shown that various factors (e.g., growth factors and mechanical stress) are responsible for the chondrogenic formation of fibroblastic cells ${ }^{22,23}$. Therefore, during the insertion development phase, undifferentiated tendon fibroblasts with the capability to form chondrocytes may migrate to the cartilage region. This remains to be clarified in a future study.

This study had several limitations. First, the findings obtained from these data may differ from findings in humans. However, the rat shoulder closely approximates the human shoulder in terms of anatomy ${ }^{13}$, and various rotator cuff tear models have been established using this rat model20,24-26. Second, we investigated Sprague-Dawley rats at approximately 16 weeks of age, and this animal model may not reflect the tendon degeneration associated with human rotator cuff tears. Finally, this study had a limited sample size, with a qualitative rather than quantitative analysis. Further quantitative analyses, over a wider age 
range, are required to compare normal and injured supraspinatus tendon-bone insertions.

In the present study, FIB/SEM tomography was used to investigate the 3D ultrastructure of cells in the normal supraspinatus tendon insertion site in rats. The study revealed distinct cells intermediate between chondrocytes and fibroblastic cells, suggesting their biomechanical significance at the insertion area. Therefore, these novel findings may contribute necessary knowledge for better regeneration of tendonto-bone insertions after rotator cuff repair.

\section{Acknowledgments}

This authors thank Mr. Ryuhei Higashi and Akinobu Togo for their technical assistance. This work was supported by the Japan Society for the Promotion of Science (JSPS) KAKENHI 7305-24791573 [Grant-inAid for Young Scientists (B)].

\section{References}

1. Rodeo SA, Arnoczky SP, Torzilli PA, Hidaka C, Warren RF Tendon-healing in a bone tunnel. A biomechanical and histological study in the dog. J Bone Joint Surg Am. 1993;75:17951803.

2. Benjamin M, Evans EJ, Copp L. The histology of tendon attachments to bone in man. J Anat. 1986;149:89-100.

3. Cooper RR, Misol S. Tendon and ligament insertion. A light and electron microscopic study. J Bone Joint Surg Am. 1970;52:1-20.

4. Shaw HM, Benjamin M. Structure-function relationships of entheses in relation to mechanical load and exercise. Scandinavian journal of medicine \& science in sports. 2007;17:303-315

5. Galatz L, Rothermich S, VanderPloeg K, Petersen B, Sandell $\mathrm{L}$, Thomopoulos S. Development of the supraspinatus tendonto-bone insertion: localized expression of extracellular matrix and growth factor genes. J Orthop Res. 2007;25:1621-1628.

6. Kjaer M. Role of extracellular matrix in adaptation of tendon and skeletal muscle to mechanical loading. Physiological reviews. 2004;84:649-698.

7. Messner K. Postnatal development of the cruciate ligament insertions in the rat knee. morphological evaluation and immunohistochemical study of collagens types I and II. Acta Anat (Basel). 1997;160:261-268.

8. Schneider H. Zur struktur der sehnenansatzzonen. Z Anat Entwicklungsgesch. 1956;119:431-456.

9. Schwartz AG, Pasteris JD, Genin GM, Daulton TL, Thomopoulos S. Mineral distributions at the developing tendon enthesis. PLoS One. 2012;7:e48630.

10. Thomopoulos S, Williams GR, Gimbel JA, Favata M, Soslowsky LJ. Variation of biomechanical, structural, and compositional properties along the tendon to bone insertion site. J Orthop Res. 2003;21:413-419.

11. Knott G, Marchman H, Wall D, Lich B. Serial section scanning electron microscopy of adult brain tissue using focused ion beam milling. The Journal of neuroscience: the official journa of the Society for Neuroscience. 2008;28:2959-2964.

12. Ohta K, Sadayama S, Togo A, Higashi R, Tanoue R, Nakamura K. Beam deceleration for block-face scanning electron microscopy of embedded biological tissue. Micron (Oxford, England : 1993). 2012;43:612-620.

13. Soslowsky LJ, Carpenter JE, DeBano CM, Banerji I, Moalli MR. Development and use of an animal model for investigations on rotator cuff disease. J Shoulder Elbow Surg. 1996;5:383-392.

14. Padulo J, Oliva F, Frizziero A, Maffulli N. Muscles, Ligaments and Tendons Journal. Basic principles and recommendations in clinical and field science research. MLTJ. 2013;4:250-252.

15. Schneider P, Meier M, Wepf R, Muller R. Serial FIB/SEM imaging for quantitative $3 \mathrm{D}$ assessment of the osteocyte lacuno-canalicular network. Bone. 2011;49:304-311.

16. Wopenka B, Kent A, Pasteris JD, Yoon Y, Thomopoulos S. The tendon-to-bone transition of the rotator cuff: a preliminary Raman spectroscopic study documenting the gradual mineralization across the insertion in rat tissue samples. Applied spectroscopy. 2008;62:1285-1294.

17. Galatz LM, Sandell LJ, Rothermich SY, Das R, Mastny A, Havlioglu N, et al. Characteristics of the rat supraspinatus tendon during tendon-to-bone healing after acute injury. J Orthop Res. 2006;24:541-550.

18. Kanazawa $\mathrm{T}$, Soejima $\mathrm{T}$, Murakami $\mathrm{H}$, Inoue $\mathrm{T}$, Katouda $\mathrm{M}$, Nagata K. An immunohistological study of the integration at the bone-tendon interface after reconstruction of the anterior cruciate ligament in rabbits. J Bone Joint Surg Br. 2006;88: 682-687.

19. Derwin KA, Codsi MJ, Milks RA, Baker AR, McCarron JA, lannotti JP. Rotator cuff repair augmentation in a canine mode with use of a woven poly-L-lactide device. J Bone Joint Surg Am. 2009;91:1159-1171.

20. Ide J, Kikukawa K, Hirose J, lyama K, Sakamoto H, Mizuta H The effects of fibroblast growth factor-2 on rotator cuff reconstruction with acellular dermal matrix grafts. Arthroscopy. 2009;25:608-616.

21. Namdari S, Baldwin K, Ahn A, Huffman GR, Sennett BJ. Performance after rotator cuff tear and operative treatment: a case-control study of major league baseball pitchers. Journal of athletic training. 2011;46:296-302.

22. Singh M, Pierpoint M, Mikos AG, Kasper FK. Chondrogenic differentiation of neonatal human dermal fibroblasts encapsulated in alginate beads with hydrostatic compression under hypoxic conditions in the presence of bone morphogenetic protein-2. J Biomed Mater Res A. 2011:98:412-424.

23. Yamakado K, Kitaoka K, Yamada H, Hashiba K, Nakamura R, Tomita K. The influence of mechanical stress on graft healing in a bone tunnel. Arthroscopy. 2002:18:82-90.

24. Beck J, Evans D, Tonino PM, Yong S, Callaci JJ. The biomechanical and histologic effects of platelet-rich plasma on rat rotator cuff repairs. Am J Sports Med. 2012;40:2037-2044.

25. Gulotta LV, Kovacevic D, Packer JD, Deng XH, Rodeo SA Bone marrow-derived mesenchymal stem cells transduced with scleraxis improve rotator cuff healing in a rat model. Am J Sports Med. 2011;39:1282-1289.

26. Galatz LM, Silva MJ, Rothermich SY, Zaegel MA, Havlioglu N, Thomopoulos S. Nicotine delays tendon-to-bone healing in a rat shoulder model. J Bone Joint Surg Am. 2006;88:2027-2034. 